\title{
Identification of Premature Senescence Cells in the Brain of the HIV-1 Transgenic Rat (HIV-TG Rat)
}

Frank Denaro ${ }^{1}$, Francesca Benedetti ${ }^{2}$, Sergio Davinelli ${ }^{3}$, Giovanni Scapagnini ${ }^{3}$, Joseph Bryant ${ }^{2}$, Davide Zella $^{2}$, and Olga Latinovic ${ }^{2}$

1. Department of Biology, Morgan State University, Baltimore, MD, USA

2. Institute of Human Virology, University of Maryland, School of Medicine, Baltimore, MD, USA

3. University of Molise, Campobasso, Italy

From the start of the AIDS epidemic, neurologic, psychological and psychomotor symptoms have been a major characteristic of HIV infection. Even with the effective current antiretroviral therapies, these symptoms continue to be observed in a subset of patients. Approximately, 30-50 percent of people living with HIV infection continue to have symptoms of HIV-associated Neurocognitive Disorder (HAND). HAND displays a range of symptoms, the most severe form is HIV-1 Associated Dementia (HAD). However, the most common presentation is Mild Neurocognitive Disorder (MND). There are multiple mechanisms by which HIV-1 can contribute to these symptoms. One important mechanism is HIV induced chronic oxidative stress [1]. We have been investigating the changes in oxidative stress in the HIV-1 TG rat $[1,2]$. Oxidative stress has been documented in many organs during HIV infection. When it occurs in the brain, it is believed to contribute to HAND. This oxidative stress can lead to altered function in cells of the brain or even cell death. Further investigations are needed to identify the cell types which are effected by oxidative stress and undergo premature senescence. The HIV-1 transgenic rat has been chosen as a model of HIV infection because it has been shown to produce measurable levels of HIV viral genes, such as gp120, Tat and Nef [2]. The products of these genes have been detected by immunohistochemistry in cells of the rat immune system. Gp-120 has also been measured in the sera and the cerebral spinal fluid. Gp-120, Tat and Nef have been found to be toxic but Gp-120, and Tat have also been shown to cause oxidative stress in cells not infected with HIV. Also, neuropathological changes have been identified in this model which correspond to areas of increased oxidative stress, such as the Basal Ganglia $[1,2]$.

We previously examined the altered expression pattern of Nrf2/HO-1 in different tissues (spleen, liver, and brain) of the HIV-1 Tg Rat. NRF2/HO-1 is a major transcription factor that can modulate redox homeostasis. One way that it can exert its influence is by regulating the antioxidant enzyme HO-1. As the HIV -1 TG rat ages, it presents with increasing AIDS related symptoms. We found that the Nrf2/HO-1 dysfunction also increases in the older animals and that evidence for senescence also increases as identified by Beta-Galactosidase (BG) enzyme histochemistry [1]. Further studies examining the HIV-1 TG rat have shown increasing neurological changes as the animal ages. This observed increase in symptoms with age is similar to the time course of the development of symptoms in humans.

In the present study we seek to identify the cell types and distribution of BG positive cells in the HIV-1 TG rat brain. The histochemical detection of BG has been used as an indicator of senescence. The method used has been described [1]. Briefly the brain and other tissues were frozen and sections were produced on the cryostate. Brain sections were produced in the coronal plane. Areas of basal ganglia, (caudate/putamen), cortical areas, hippocampus and brain stem were investigated. These sections were then post-fixed $1 \%$ formaldehyde washed in buffer and stored at $-80 \mathrm{C}^{\circ}$ until processed for BG activity. 
HIV-1 TG Spleen was used as a positive control. Both old and young animals, and non-HIV TG Rats (negative control) were used [1].

Histochemical analysis of the HIV-TG rat brain revealed that senescent cells can be found in areas important for sensory motor processing such as the Basal Ganglia. They are also found in areas important for memory and learning which include the cortex and the hippocampus. While double labels should be done to confirm cell type, the general morphology has given some insight. Pyramidal shaped cells in the cortex suggest neurons. While the rounder cells in the Caudate/putamen may be neurons or glia. It is also possible that there can be positive WBS. The exact mechanisms of HAND continue to be under investigation, but there is growing evidence for the role of oxidative stress and free radicals in HAND [3].

\section{References:}

[1] S. Davinelli et al, Biogerontology, 15 (2014), p. 449.

[2] W. Reid et al, Proceedings of the National Academy of Science 98(16) (2001), p. 9271.

[3] The authors acknowledge funding from Grant numbers: 5UL1GM118973, 1 R29 NS31857-01A1.

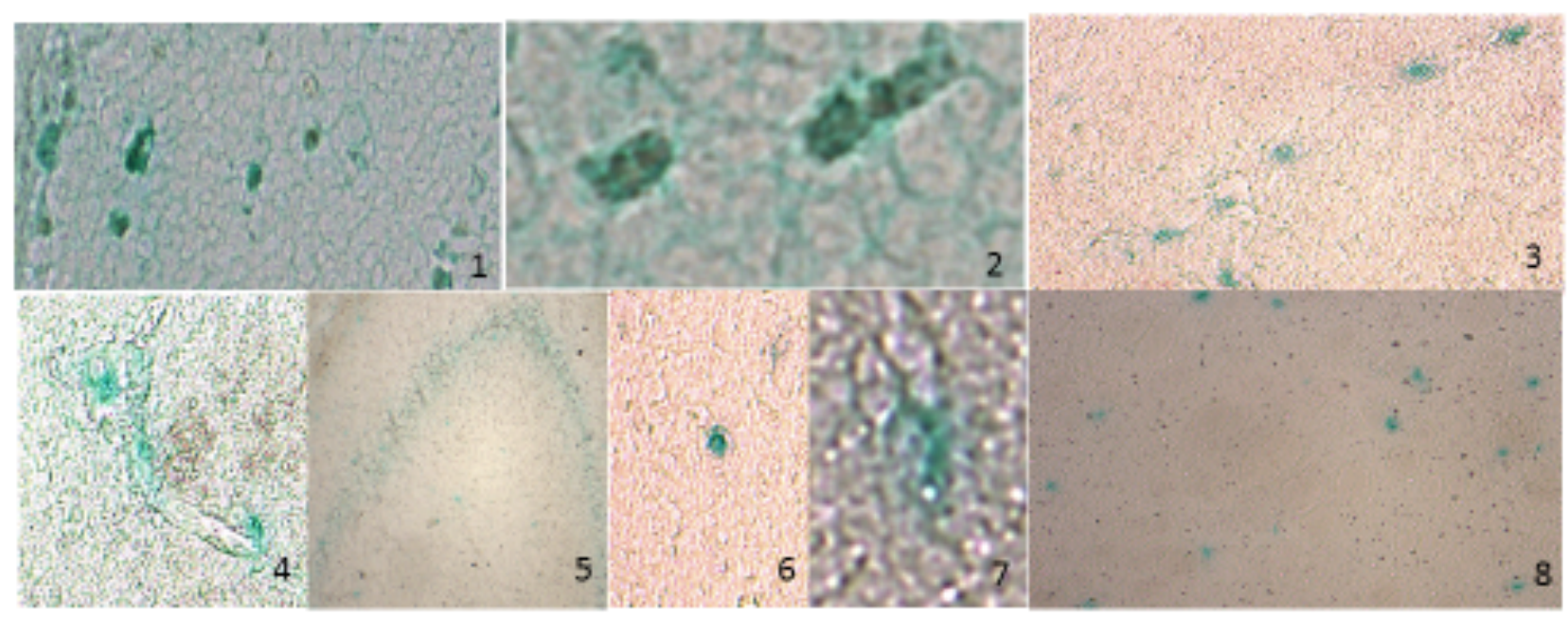

Figure 1. Beta-Galactosidase Enzyme Histochemistry HIV-1 TG Rat. BG positive cells are in green. 1.) Spleen 10X. The BG histochemistry reveals a number of different cell types in both the red and white pulp area. 2.) Spleen 60X. Examination of cells reveal that some include Hemosiderin, which suggests that these cells are macrophages. 3. and 8. ) Brain 40X. Positive cells in the caudate/ putamen. Cells can be found in isolation or groups. Some have neural morphology while others may be astrocytes. 4). Brain $60 \mathrm{X}$. BG positivity associated with blood vessels. 5). Brain 10X. The Hippocampus displays two types of staining, a very light stain and also some dark staining of individual cells. 6.) Brain 40X. Positive cells in the cortex. 7.) Brain 60X, positive cell in the cortex. 